\title{
EMBOLIA PERIFÉRICA SECUNDÁRIA À TROMBO DE TRONCO BRAQUIOCEFÁLICO: RELATO DE CASO
}

\section{RELATO DE CASO}

SANTOS, Henrique Amorim ${ }^{1}$

SANTOS, Isabella Amorim ${ }^{2}$

ARGONDIZZI, Roberto Oliveira ${ }^{3}$

GARCEZ, Michelle Maia ${ }^{4}$

REIS, Mateus Fernandes Alves dos ${ }^{5}$

FERREIRA, Amanda Karolyne Batista ${ }^{6}$

SIMONI, Anderson Lubito ${ }^{7}$

\footnotetext{
${ }_{1}^{1}$ Atualmente médico residente Cirurgia Geral Universidade Federal Triângulo Mineiro, médico graduado pela Faculdade de Ciências da Saúde de Barretos Dr Paulo Prata em 2018.
}

${ }^{2}$ Atualmente graduanda de Medicina pela Universidade de Franca.

${ }^{3}$ Atualmente graduando de Medicina pela Universidade Federal do Triângulo Mineiro.

${ }^{4}$ Atualmente graduando de Medicina pela Universidade Federal do Triângulo Mineiro.

${ }^{5}$ Atualmente graduando de Medicina pela Universidade Federal do Triângulo Mineiro.

${ }^{6}$ Atualmente médica residente Cirurgia Geral Universidade Federal Triângulo Mineiro, médica graduada pela Universidade de Uberaba em 2018.

${ }^{7}$ Médico formado pela Universidade de Uberaba (2009), Residência em Cirurgia Geral na Faculdade de Medicina de São José do Rio Preto (2011), Residência em Cirurgia Vascular na Faculdade de Medicina de São José do Rio Preto (2013) e Residênica em Angioradiologia e Cirurgia Endovascular na Faculdade de Medicina de São José do Rio Preto, FAMERP, São Jose Do Rio Preto (2014), Brasil. 
SANTOS, Henrique Amorim. Et al. Embolia periférica secundária à trombo de tronco braquiocefálico: Relato de caso. Revista Científica Multidisciplinar Núcleo do Conhecimento. Ano 05, Ed. 09, Vol. 06, pp. 96-106. Setembro de 2020. ISSN: 2448-0959, Link de acesso: https://www.nucleodoconhecimento.com.br/saude/embolia-periferica

\section{RESUMO}

O tromboembolismo arterial de membros superiores é menos frequente que o de membros inferiores, e por isso, muitas vezes subdiagnosticado. Contudo, trata-se de uma emergência clínica e o atraso no diagnóstico e no tratamento podem resultar em isquemia e grave comprometimento funcional, cerebral e cardiológico. Desse modo, o presente trabalho tem a finalidade de descrever um relato de caso clínico de embolia de artérias ulnar e radial devido à trombose de artéria subclávia fornecendo as informações necessárias para que o médico reconheça essa condição, possibilitando o diagnóstico e o tratamento precoces, bem como a identificação e a correção dos fatores de risco.

Palavras-chave: embolia arterial periférica, trombose arterial, doença da artéria subclávia.

\section{INTRODUÇÃO}

A Oclusão Arterial Aguda (OAA), é uma emergência vascular caracterizada pela perda repentina de perfusão de determinada região, por até 2 semanas após algum evento desencadeante. A OAA pode ocorrer devido à oclusão tromboembólica, traumas penetrantes ou contusos. O principal local de OAA periférica é a artéria femoral superficial (SMITH; LILIE, 2020). Dessa forma, os eventos tromboembólicos de membros superiores são mais raros que os de membros inferiores, correspondendo a cerca de 16 a 32\% dos casos de embolia arterial periférica (WOLOSKER et. al., 1996).

O tromboembolismo do membro superior está relacionado à eventos cardíacos, sendo a fibrilação atrial a principal etiologia cardíaca, e não cardíacos, afetando 
principalmente a população idosa devido à presença de comorbidades (CHISARI et. al., 2016). Deste modo, apesar de raros, esses eventos devem ser reconhecidos rapidamente, pois o atraso no diagnóstico e no tratamento pode implicar em isquemia da região, levando a complicações graves.

Assim, o objetivo deste trabalho é relatar um caso de embolia de artérias radial e ulnar, devido à trombose de artéria subclávia, admitido no Hospital das Clínicas da Universidade Federal do Triângulo Mineiro (HC-UFTM), abrangendo o diagnóstico e o tratamento do caso, para colaborar com o desenvolvimento do pensamento clínico e com a finalidade de auxiliar na identificação precoce de casos de tromboembolismo de membros superiores.

\section{RELATO DE CASO}

Paciente do sexo feminino, 57 anos, natural e procedente de Sacramento, admitida no pronto socorro da sua cidade de origem devido à cianose distal em mão direita e dor súbita de forte intensidade em membro superior direito (MSD), não relacionada à trauma ou outro evento desencadeante, onde permaneceu internada por cinco dias até ser transferida para o HC-UFTM.

$\mathrm{Na}$ admissão ao HC-UFTM, a paciente ainda apresentava dor de forte intensidade, e, ao exame físico, apresentava paresia e parestesia em MSD, mão direita com cianose distal fixa, fria, edemaciada, com perfusão lentificada e ausência de pulsos radial e ulnar, sendo elencada a hipótese diagnóstica de embolia em artérias radial e ulnar direita. A paciente negava qualquer acometimento vascular prévio, como trombose venosa profunda, tromboembolismo pulmonar, infarto agudo do miocárdio ou mesmo acidente vascular encefálico. Relatava ter feito uso de anticoncepcional oral combinado (ciclo 21) por 10 anos, interrompendo o uso aos 51 anos. Ademais, a paciente relatava ser tabagista, com carga tabágica de 60 maços/ano, hipertensa e negava etilismo. Eletrocardiograma da admissão possuía ritmo cardíaco sinusal.

Dessa forma, foi realizado ultrassonografia com doppler de MSD, no qual foi evidenciado fluxo de padrão monofásico em terço distal da artéria radial e em terço 
médio da artéria ulnar, além de presença de material hiperecóico compatível com trombo no interior da artéria ulnar, em seu terço distal, a cerca de $3 \mathrm{~cm}$ da face palmar, obstruindo o fluxo arterial da região. Isto posto, a paciente foi encaminhada para cirurgia de tromboembolectomia de urgência, com uso Fogarty, com fibrinólise intra arterial e devido risco de síndrome compartimental em tal caso, fasciotomia descompressiva em antebraço e mão à direita (figuras 1, 2 e 3). Após 2 dias do procedimento cirúrgico, a paciente apresentou melhora da paresia e da hipoestesia, porém com persistência da cianose fixa de extremidade direita, sendo indicado ecocardiograma (ECO), para avaliação cardíaca e pesquisa da origem dos êmbolos, contudo não foi evidenciado nenhuma alteração, com ausência de trombos ou de vegetação.

Figura 1 - Palma da mão no pós operatório imediato

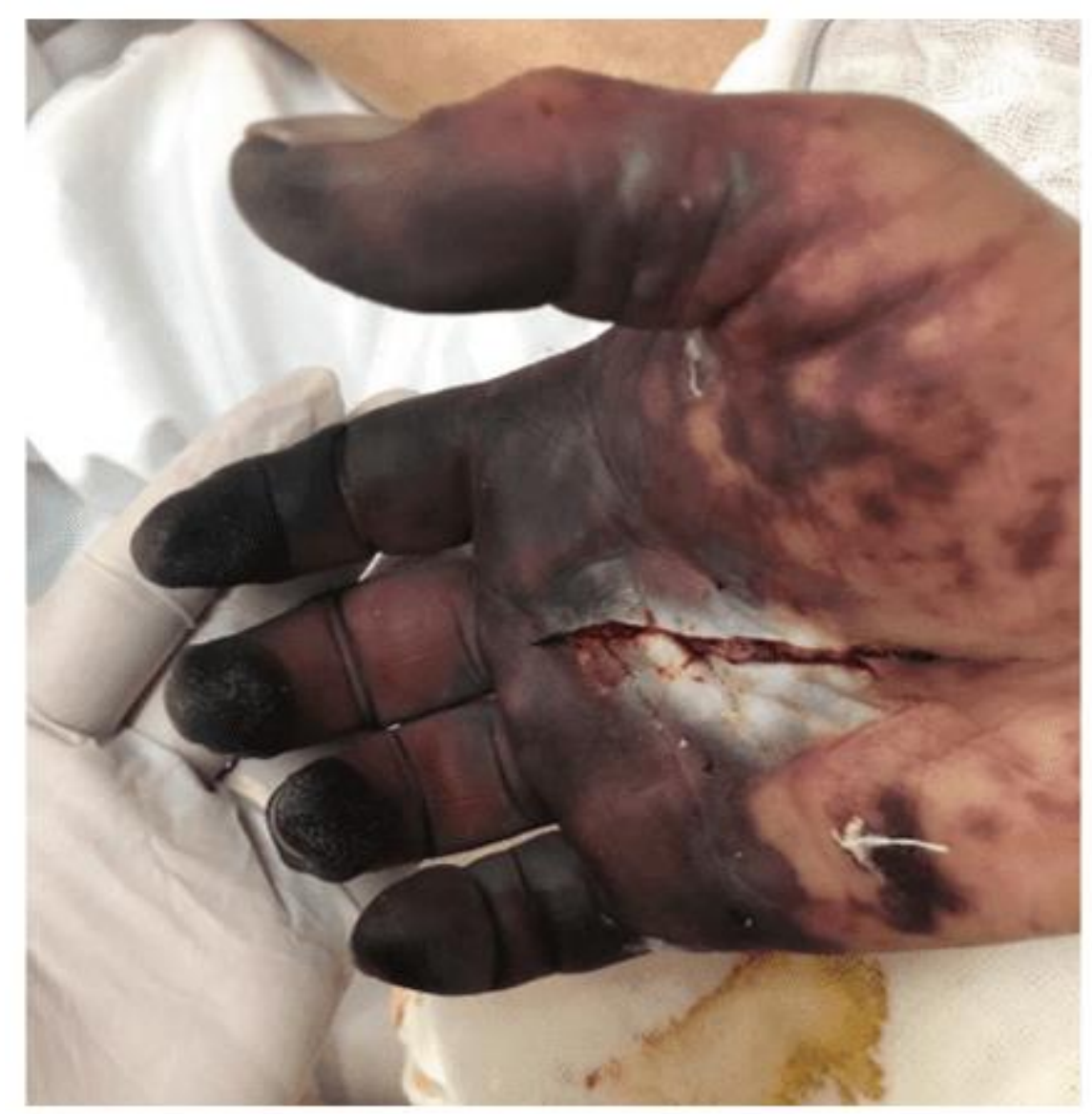

Fonte: Arquivo Pessoal 
Figura 2 - Dorso da mão no pós operatório imediato

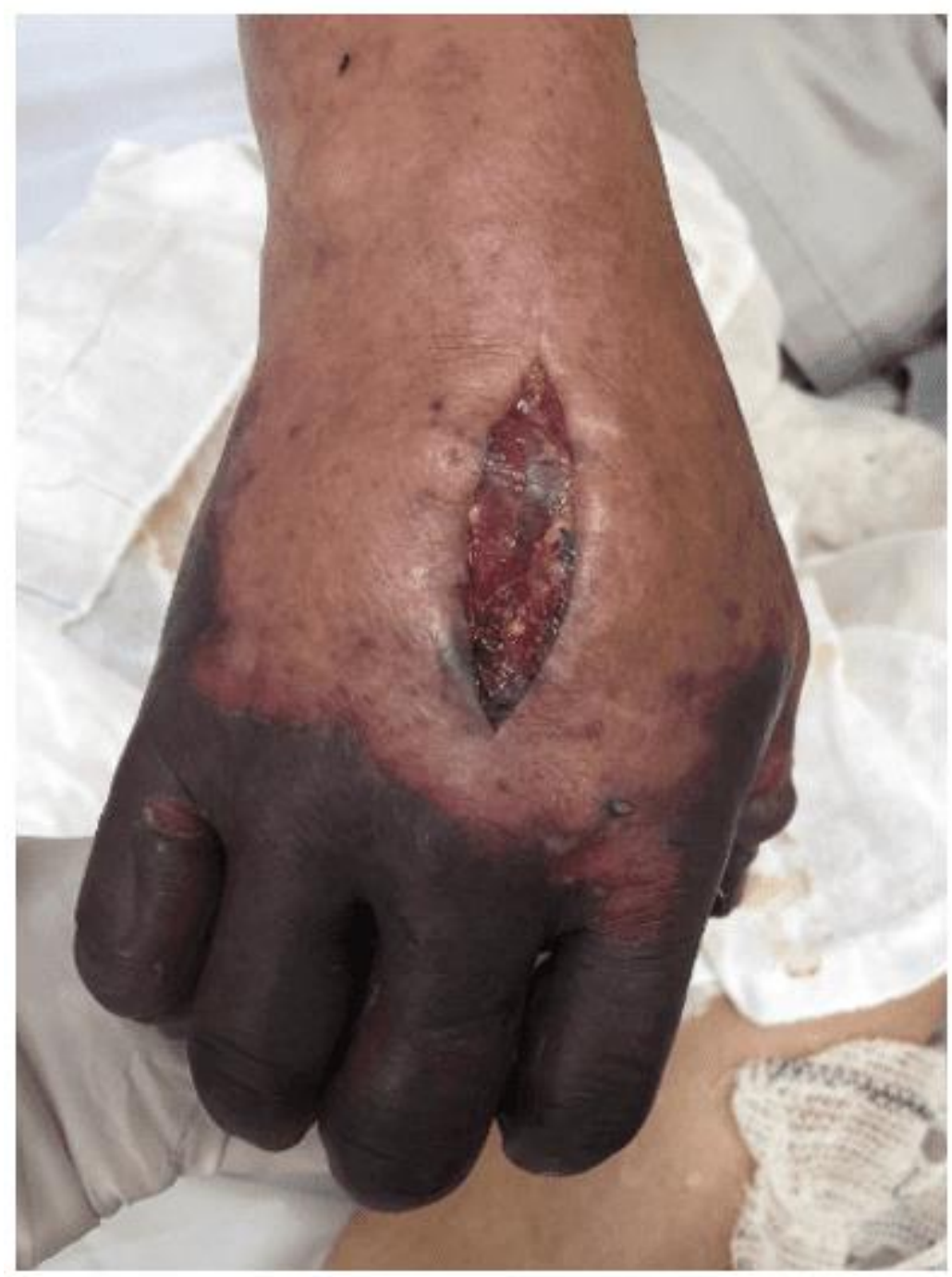

Fonte: Arquivo Pessoal 
Figura 3 - Mão e antebraço no pós operatório imediato

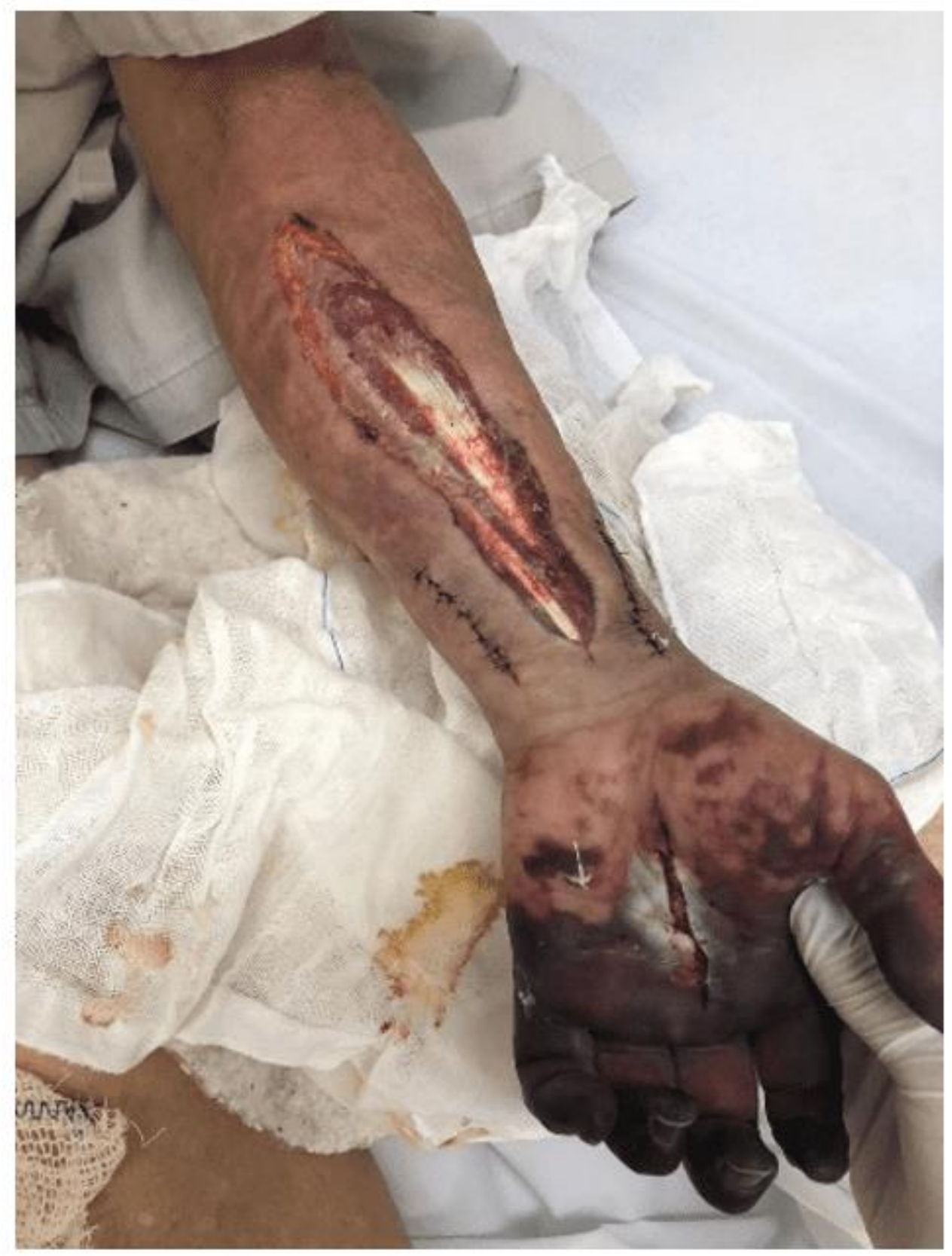

Fonte: Arquivo Pessoal

Posteriormente, no sexto dia de pós operatório, a paciente apresentou piora do quadro, com pulsos radial e ulnar à direita filiformes, paresia e hipoestesia em mão direita, além de necrose seca em quirodáctilos direitos, sem sinais de perfusão. No nono dia de pós-operatório, devido à piora na evolução do quadro de necrose 
isquêmica de quirodáctilos à direita, foi optado por amputação transmetacarpiana. Desse modo, a paciente foi encaminhada para cirurgia de amputação, com preservação do primeiro quirodáctilo (figura 4). Após o procedimento cirúrgico, a paciente evoluiu bem, apenas com relato de dor de leve intensidade no coto cirúrgico.

Figura 4 - Pós operatório da amputação

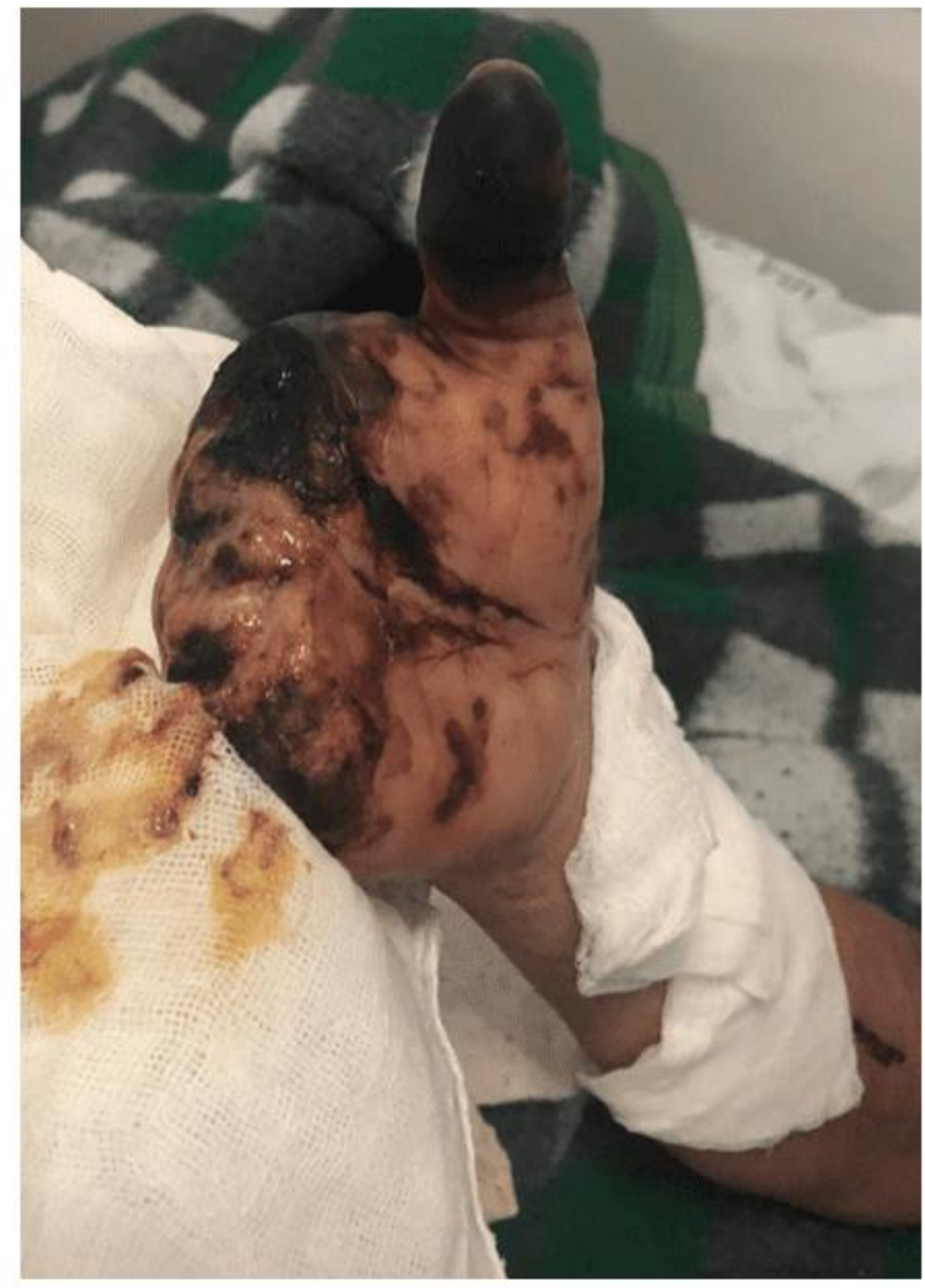

Fonte: Arquivo Pessoal 
Um dia após a amputação transmetacarpiana, foi solicitado angiotomografia computadorizada de tórax e membro superior direito, para pesquisa da origem dos êmbolos, no qual evidenciou-se um trombo alongado de $2,4 \mathrm{~cm}$ parcialmente aderido no tronco braquiocefálico a $0,7 \mathrm{~cm}$ de sua origem, apresentando certa mobilidade e ocupando $40 \%$ do lúmen vascular do local, sendo proposta cirurgia de trombectomia com via de acesso por esternotomia após duas semanas (figuras 5 e 6). O procedimento cirúrgico ocorreu sem intercorrências, sendo realizado toracotomia longitudinal mediana, esternotomia e abertura do saco pericárdico, seguido de dissecção anatômica dos grandes vasos e infusão de heparina 5000ui EV. Em seguida, realizou-se o clampeamento das artérias carótidas, subclávia e tronco braquiocefálico, seguido de arteriotomia do tronco e retirada da placa lipídica mole da íntima da artéria. Procedeu-se com sutura da artéria com fio prolene 5-0, seguido de fechamento do saco pericárdio e esternorrafia com fio de aço. Por fim, realizou-se fechamento do subcutâneo com fio vicryl 3-0 e finalizou o procedimento com sutura intradérmica com fio monocryl 5-0.

Figura 5 - Angiotomografia de tórax

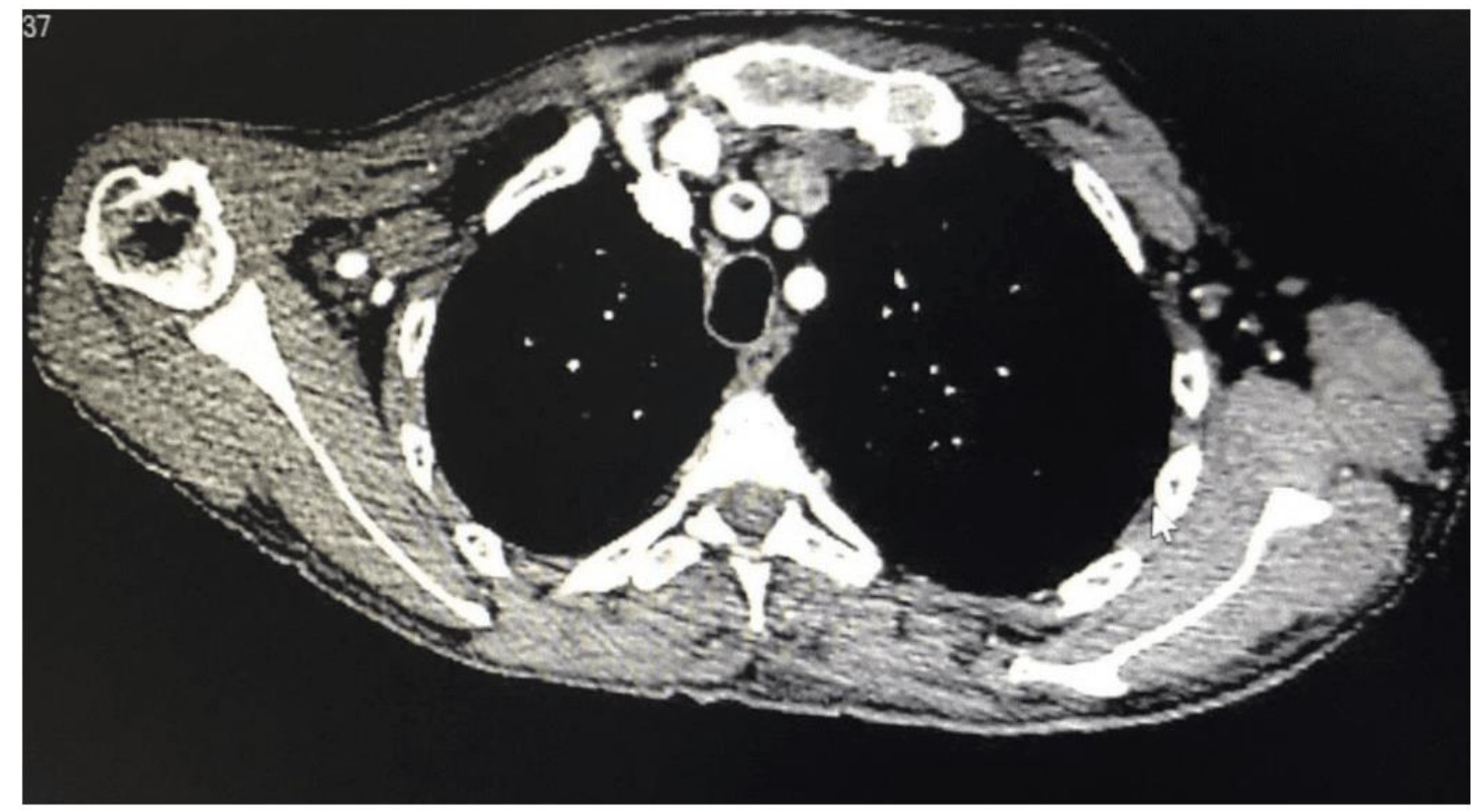

Fonte: Arquivo Pessoal 
Figura 5 - Angiotomografia de tórax

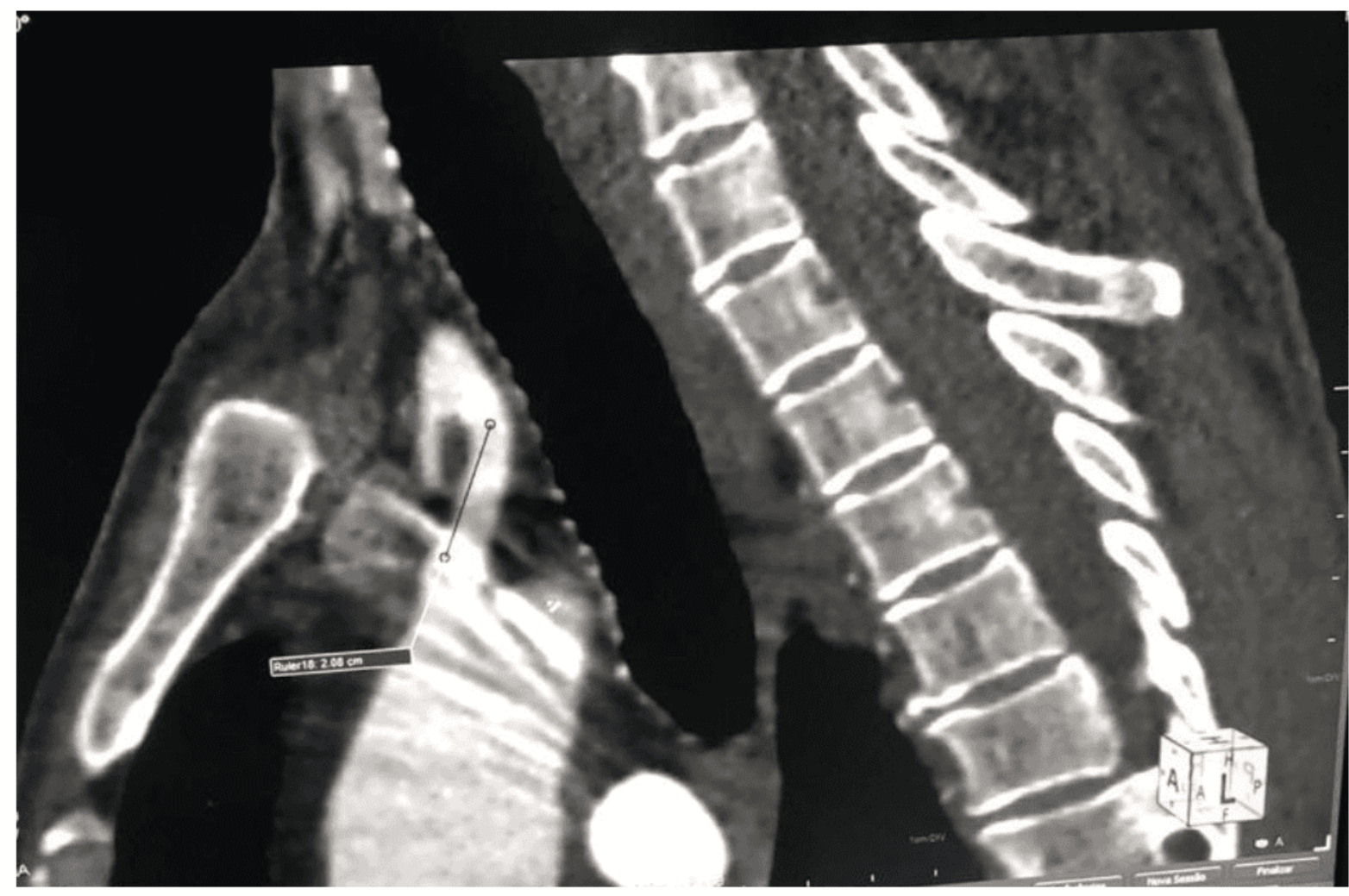

Fonte: Arquivo Pessoal

A paciente não apresentou complicações no pós operatório, relatando apenas queixas álgicas de leve intensidade na região da ferida cirúrgica e recebeu alta hospitalar uma semana após o último procedimento cirúrgico, sendo indicado o acompanhamento no ambulatório de cirurgia vascular para a avaliação do primeiro quirodáctilo.

\section{DISCUSSÃO}

A isquemia de membros superiores é menos comum, com relação à de membros inferiores, com relativamente poucos casos relatados. No entanto, atrasos no diagnóstico e no tratamento podem resultar em grave comprometimento funcional e incapacidade, mesmo na ausência de perda evidente de tecido (BAE et. al., 2015). Em um contexto clínico, o ombro e o cotovelo são mais tolerantes à isquemia, devido à sua circulação colateral bem desenvolvida, desse modo, é mais comum observar sintomas isquêmicos abaixo do cotovelo (BAE et al, 2015). Isso é constatado na 
paciente, visto à sintomatologia prevalente na extremidade de membro superior direito, associada ao território de irrigação de artérias radial e ulnar.

Com relação à oclusão arterial aguda de etiologia tromboembólica - mais comum - a maioria dos êmbolos se origina no coração, associados à fibrilação atrial, infarto do miocárdio recente e doença valvar. A oclusão embólica associada à fibrilação atrial, ou outras fontes, é classicamente vista na artéria braquial antes da bifurcação das artérias radial e ulnar (BAE et. al., 2015). Entretanto, nesse caso, a paciente não apresentou alterações ao ECO, sendo uma oclusão embólica não relacionada a causas cardíacas.

Além disso, placas ateroscleróticas também podem se desenvolver dentro das artérias de membros superiores e gerar trombose aguda resultante da erosão ou ulceração da placa, assim como na paciente relatada, no qual através da angiotomografia, foi possível identificar a presença de um trombo móvel na artéria subclávia. Contudo, a paciente apresentava fatores de risco importantes para doença vascular periférica, como hipertensão, tabagismo e dislipidemia.

Com relação à apresentação clínica, o tromboembolismo de membro superior é caracterizado por uma diminuição repentina na perfusão do membro que ameaça sua viabilidade. Os sintomas, que se desenvolvem ao longo de um período de horas, incluem dor, parestesia, paresia e paralisia do membro afetado. Os achados físicos podem incluir ausência de pulsos distais à oclusão, pele fria e pálida, sensibilidade e força reduzidas (CHISARI et. al., 2016).

A presença de fluxo, nas artérias radial e ulnar da extremidade sintomática, deve ser avaliada rotineiramente com instrumento de Doppler. Avaliação de sensibilidade, força e pulsos por Doppler, podem ser usados para estadiar a gravidade da isquemia e para avaliar se o membro é viável, ameaçado ou irreversivelmente danificado (CHISARI et. al., 2016).

Os diagnósticos diferenciais mais relevantes a serem aventados, com relação à oclusão arterial aguda, associada à evento tromboembólico, são: isquemia crônica de 
membro secundária à aterosclerose e causas não ateroscleróticas, isquemia vasoespástica do membro, trombose venosa profunda extensa de membro superior, síndrome compartimental aguda e dor aguda não isquêmica nos membros, secundária a ataque agudo de gota, neuropatia, lesão traumática e hemorragia espontânea (CHISARI et. al., 2016).

O tratamento de escolha para isquemia aguda devido à quadro embólico do membro superior é a correção cirúrgica por embolectomia usando um cateter balão Fogarty (BAE et. al., 2015). Segundo Chisari (2016), é descrito um risco maior de tromboembolectomia em mulheres, sendo apontadas algumas explicações, entre elas, o menor diâmetro das artérias nas mulheres em relação aos homens. Assim, êmbolos menores podem ser grandes o suficiente para ocluir a artéria braquial nas mulheres, enquanto nos homens eles podem migrar para a artéria radial ou ulnar e ser clinicamente assintomáticos, devido à anastomose das artérias pré-braquiais através da arcada arterial da mão, correspondendo com o caso apresentado, por se tratar de uma paciente do sexo feminino.

Com relação ao período pós operatório, a restauração de pulsos arteriais distais palpáveis, sinais audíveis de Doppler arterial e melhora visível da perfusão (por exemplo, aumento da temperatura da pele e produção de suor) sugerem sucesso do tratamento (CHISARI et. al., 2016). A síndrome compartimental, caracterizada por um aumento dramático na pressão compartimental, representa a complicação mais temível após a reperfusão. Além disso, pacientes com tromboembolismo de membro superior apresentam risco aumentado de amputação de membro superior, acidente vascular encefálico e morte (CHISARI et. al., 2016). Na paciente, devido à evolução da necrose de dedos da mão direita, foi indicada cirurgia de amputação transmetacarpiana de mão direita, com preservação do primeiro dedo.

Nesse sentido, em estudo dinamarquês de Andersen et. al. (2010) com quase 1400 pacientes submetidos à tromboembolectomia, relataram uma taxa de amputação de $2,2 \%$ em homens e 3,6\% em mulheres. O maior risco de amputação entre as mulheres também persistiu por um longo período em comparação com os homens, com risco 
aumentado de amputação por 5 anos em mulheres e por 1 ano em homens (ANDERSEN et. al., 2010).

\section{CONSIDERAÇÕES FINAIS}

A oclusão da artéria subclávia, embora rara quando comparada a oclusão em membros inferiores, deve ser lembrada, já que pode acarretar em quadros isquêmicos que afetam não somente o membro superior, mas também podem acometer o cérebro e, mais raramente, o coração (MUBARIK; IQBAL, 2020). Embora, nesse caso, a paciente não apresentasse sintomas neurológicos, houve comprometimento grave do membro, tendo como consequência a amputação do segundo ao quinto quirodáctilo. Segundo Ochoa e Yeghiazarians (2011), o tratamento endovascular possui eficácia comprovada e morbidade e mortalidade diminuídas. Entretanto, nesse caso, por se tratar de um tromboembolismo flutuante, a melhor abordagem foi a cirúrgica.

Nesse contexto, o médico deve conseguir identificar o evento tromboembólico em membro superior, para possibilitar o diagnóstico e o tratamento precoces, a fim de evitar possíveis complicações (OCHOA; YEGHIAZARIANS, 2011). Além disso, é importante estar ciente das recomendações de triagem, que incluem pacientes em processo de revascularização da artéria coronária utilizando a artéria intermamária, a alta suspeita de estenose subclávia e pacientes com histórico de doença arterial periférica ou diferenças de pressão arterial sistólica em seus membros superiores de mais de $10 \mathrm{mmHg}$ (MUBARIK; IQBAL, 2020).

Ademais, por se tratar de um evento tromboembólico, onde os fatores de risco incluem distúrbios metabólicos, é essencial o acompanhamento com uma equipe multidisciplinar, visando a redução dos fatores de risco como o tabagismo, hipertensão, dislipidemia e diabetes.

\section{REFERÊNCIAS}

ANDERSEN, Ljubica Vukelic; MORTENSEN, Leif Spange; LINDHOLT, Jes; FAERGEMAN, Ole; HENNEBERG, Eskild Winther; FROST, Lars. Upper-limb 
thrombo-embolectomy: national cohort study in Denmark. Eur J Vasc Endovasc Surg, 2010; 40: 628-34, 2010.

BAE, Miju et al. Upper limb ischemia: clinical experiences of acute and chronic upper limb ischemia in a single center. The Korean journal of thoracic and cardiovascular surgery, v. 48, n. 4, p. 246, 2015.

CHISARI, Alberto et al. Upper limb ischemia from arterial thromboembolism: a comprehensive review of incidence, etiology, clinical aspects, diagnostic tools, treatment options and prognosis. Minerva Cardioangiol, v. 64, n. 625, p. e34, 2016.

MUBARIK, Ateeq; IQBAL, Arshad Muhammad. Subclavian Artery Thrombosis. StatPearls [Internet], Treasure Island (FL): StatPearls Publishing, 2020 Jan. Disponível em: https://www.ncbi.nlm.nih.gov/books/NBK537275/. Acesso em 03/09/2020.

OCHOA, Victor M; YEGHIAZARIANS, Yerem. Subclavian artery stenosis: A review for the vascular medicine practitioner. Vascular Medicine, 2011;16(1):29-34, 2011.

SMITH, David A; LILIE, Craig J. Acute Arterial Occlusion. StatPearls [Internet], Treasure Island (FL): StatPearls Publishing, 2020 Jan. Disponível em: https://www.ncbi.nlm.nih.gov/books/NBK441851/. Acesso em 03/09/2020.

WOLOSKER, Nelson et al. Embolia de Membros Superiores. Cir. vasc. Angiol, 12(3):120-4, set. 1996.

Enviado: Setembro, 2020.

Aprovado: Setembro, 2020. 